\title{
CD38 gene-modified dendritic cells inhibit murine asthma development by increasing IL-12 production and promoting Th1 cell differentiation
}

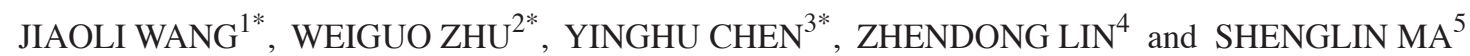 \\ ${ }^{1}$ Department of Respiratory Medicine, Nanjing Medical University, Affiliated Hangzhou Hospital \\ (Hangzhou First People's Hospital), Hangzhou, Zhejiang 310006; ${ }^{2}$ Department of Cardiology, The First Affiliated Hospital, \\ Zhejiang University School of Medicine, Hangzhou, Zhejiang 310003; ${ }^{3}$ Division of Infection Disease, \\ Zhejiang Key Laboratory for Neonatal Diseases, Children Hospital, Zhejiang University School of Medicine, Hangzhou, \\ Zhejiang 310006; ${ }^{4}$ Department of Gynecology and Obstetrics, The Second Affiliated Hospital, Wenzhou Medical University, \\ Wenzhou, Zhejiang 325035; ${ }^{5}$ Department of Oncology, Hangzhou First People's Hospital, \\ Hangzhou Hospital Affiliated to Nanjing Medical University, Hangzhou, Zhejiang 310006, P.R. China
}

Received September 20, 2015; Accepted August 31, 2016

DOI: $10.3892 / \mathrm{mmr} .2016 .5756$

\begin{abstract}
Predominant T helper (Th)2 and impaired Th1 cell polarization has a crucial role in the development of asthma. Cluster of differentiation (CD)38 is associated with the increased release of interleukin (IL)-12 from dendritic cells (DCs) and DC-induced Th1 cell polarization. However, whether CD38 expression affects DC function in asthma development remains unknown. In the current study, adenoviruses were constructed containing the murine CD38 gene. Overexpression of CD38 protein level in DCs induced from bone-marrow derived DCs (BMDCs) by recombinant mouse granulocyte macrophage colony-stimulating factor and IL-4 was achieved through $24 \mathrm{~h}$ adenovirus infection. The results demonstrated that BMDCs with CD38 overexpression exhibited no phenotypic change; however, following stimulation with lipopolysaccharide (LPS), maturation and IL-12 secretion were increased. In addition, CD38-overexpressing BMDCs stimulated with LPS exhibited more effective Th1 cell differentiation. Mice that were administered CD38-overexpressing
\end{abstract}

Correspondence to: Dr Shenglin Ma, Department of Oncology, Hangzhou First People's Hospital, Hangzhou Hospital Affiliated to Nanjing Medical University, 261 Huansha Road, Hangzhou, Zhejiang 310006, P.R. China

E-mail: mashenglin@medmail.com.cn

*Contributed equally

Abbreviations: DCs, dendritic cells; BMDCs, bone marrow-derived DCs; Ad-CD38, CD38 adenovirus; Ad-LacZ, LacZ adenovirus; BALF, bronchoalveolar lavage fluid; FACS, fluorescence-activated cell sorting

Key words: CD38, dendritic cells, asthma, Th1 polarization, adenovirus
BMDCs exhibited milder symptoms of asthma. Furthermore, decreased IL-4, IL-5 and IL-13 levels were detected in bronchoalveolar lavage fluid (BALF), reduced immunoglobulin E levels were measured in the sera, and increased interferon- $\gamma$ was detected in BALF from the recipients of CD38-overexpressing BMDCs. Increased phosphorylated-p38 expression was also detected in LPS-stimulated CD38-overexpressing BMDCs, whereas pretreatment with a $\mathrm{p} 38$-specific inhibitor was able to abolish the effects of LPS stimulation and CD38 overexpression on IL-12 release and Th1 cell differentiation in BMDCs. These results suggested that CD38 may be involved in the DC function of alleviating asthma via restoration of the Th1/Th2 balance, thus providing a novel strategy for asthma therapy.

\section{Introduction}

The incidence of asthma remains high worldwide. As a chronic airway inflammatory disease, asthma not only harms patient's physical and mental health, but is also considered a burden to society $(1,2)$. There are several clinical interventions used to treat asthma, including traditional treatment with glucocorticoids; however, the effects of treatment are not always satisfactory. Glucocorticoid therapy is an effective treatment for asthma; however, its side effects greatly constrain clinical applications (3). Therefore, it is necessary to explore novel therapeutic strategies for the treatment of asthma.

Various cells, including eosinophils, basophils and cluster of differentiation (CD) $4^{+} \mathrm{T}$ cells, and several proinflammatory mediators, such as tumor necrosis factor- $\alpha$ and interleukin (IL)-17, are involved in the chronic respiratory inflammatory responses of asthma $(4,5)$. However, the mechanism underlying asthma remains largely unknown. $\mathrm{CD}^{+} \mathrm{T}$ cells are critical cellular mediators of asthma and inflammation. The $\mathrm{CD}^{+}$ $\mathrm{T}$ cell subset can be divided into T helper (Th) 1 and Th2 cells, according to the type of cytokines they release and their function (6). The impaired balance of Th1/Th2, and the abnormal polarization of Th2 are considered the most important 
mechanisms of asthma (7). During the development of asthma, Th2 cells orchestrate the inflammatory microenvironment via the production of Th2 cytokines (IL-4, IL-5 and IL-13), thus contributing to the pathological process of asthma (8). The balance between Th1 and Th2 cells is finely tuned and their differentiation is reciprocally inhibited (9); therefore, increased Th1 polarization is important for the control of asthma.

Dendritic cells (DCs) are the most important antigen-presenting cells; they are able to present antigens to naïve $\mathrm{CD}^{+} \mathrm{T}$ cells, and mediate their activation and differentiation (10). In the pulmonary system, DCs predominantly exist in the bronchial epithelium, subepithelial tissue and bronchial lymph nodes, where they exhibit a grid-like distribution. DCs cluster with T cells in the subepithelial tissue and finely orchestrate the polarization of Th cells, which serves a key role in the regulation of inflammation and airway tolerance $(11,12)$. It has previously been reported that following injection of ovalbumin (OVA) into the airway, DCs can activate naïve $\mathrm{CD}^{+} \mathrm{T}$ cells surrounding the pulmonary lymph nodes to a Th2 phenotype, thus inducing asthma (13). The release of IL-12 from DCs has a central role in the induction of Th1 cell differentiation and IL-12-dependent DC-induced Th1 cell differentiation may suppress murine asthma $(14,15)$. Decreased IL-12 levels are involved in Th2 polarization and are associated with the severity of asthma (16). CD38, which consists of 304 amino acids, is a single chain type 2 transmembrane glycoprotein that belongs to the multifunctional ectoenzyme family $(17,18)$. CD38 is expressed in several types of cells, including T cells, B cells and monocytes $(19,20)$. Furthermore, as a multifunctional ectoenzyme, CD38 possesses numerous immunologically relevant functions. For example, CD38 participates in T-cell activation, B-cell growth and prevents apoptosis of tonsillar germinal center B cells (21). Compared with wild type mice, the splenocytes from CD38-knockout mice were shown to secrete reduced interferon (IFN) $-\gamma$ and increased IL-4, thus suggesting that CD38 has a role in Th1 polarization (22). In addition, it has been reported that $\mathrm{CD} 38$ expression fluctuates during differentiation of human monocyte-derived DCs. CD38 was downregulated during differentiation into immature monocyte-derived DCs, whereas expression was restored upon maturation. When CD38 signaling was suppressed, monocyte-derived DCs exhibited a more immature phenotype and a reduced ability to present antigens and produce IL-12 (23).

Due to the key role of IL-12 in the polarization of Th1 cells, the present study aimed to determine whether overexpression of CD38 in DCs was able to improve IL-12 production and Th1 cell polarization. Furthermore, the effects of CD38 overexpression in DCs on asthma development were determined.

\section{Materials and methods}

Reagents. Recombinant mouse granulocyte macrophage colony-stimulating factor (rmGM-CSF) and IL-4 were purchased from PeproTech (Rocky Hill, NJ, USA). Mouse Naïve CD4 ${ }^{+}$ $\mathrm{T}$ cell Isolation kit was obtained from Miltenyi Biotec $\mathrm{GmbH}$ (Bergisch Gladbach, Germany). Anti-mouse IL-4 (BE0045) antibodies were purchased from Bio X Cell (West Lebanon, NH, USA). Anti-mouse major histocompatibility complex class II (MHC-II) (107607), anti-mouse CD80 (104707), anti-mouse CD86 (105007) and anti-mouse CD40 (124609) antibodies were purchased from Biolegend (San Diego, CA, USA). Anti-mouse CD38 (90), anti-mouse CD4 (17-0041), anti-mouse IFN- $\gamma$ (11-7311) antibodies; mouse IL-4(80-7044-22),IL-5(80-7054-22), IL-13 (88-7137-22), IFN- $\gamma$ (88-7314-22) and immunoglobulin (Ig)E (88-50460-22) enzyme-linked immunosorbent assay (ELISA) kits; and the Intracellular Fixation \& Permeabilization Buffer Set were purchased from eBioscience (San Diego, CA, USA). Anti-phosphorylated (p)-p38 (sc-7973) and p38 (sc-7972) antibodies, and the p38-specific inhibitor SB203580 $\left(10 \mu \mathrm{g} / \mathrm{ml}\right.$ at $37^{\circ} \mathrm{C}$ for $\left.30 \mathrm{~min}\right)$ were purchased from Santa Cruz Biotechnology, Inc. (Dallas, TX, USA). Lipopolysaccharide (LPS) from Escherichia coli and OVA were purchased from Sigma-Aldrich (Merck Millipore, Darmstadt, Germany).

Mice. For all experiments, female mice were used. A total of 15 mice were used to induce BMDCs, 25 to isolate splenocytes and 72 to establish the asthma model. Female C57BL/6J mice (age, 6-8 weeks; weight, 18-20 g) were purchased from Joint Ventures Sipper BK Experimental Animal Co., Ltd. (Shanghai, China). Mice were maintained in specific pathogen-free facilities with temperature ranging from $22-24^{\circ} \mathrm{C}$, humidity ranging from $50-60 \%$ and $12 \mathrm{~h}$ of light/dark cycle at Zhejiang University (Hangzhou, China). Mice had free access to food and water and were sacrificed by intraperitoneal injection with nembutal (160 mg/kg; Sigma-Aldrich; Merck Millipore). All experiments using mice were approved by and performed according to the guidelines of the Animal Ethics Committee of Zhejiang University.

Construction of recombinant mouse CD38 adenovirus (Ad-CD38). Ad-CD38 was constructed using the AdMax ${ }^{\mathrm{TM}}$ system (Microbix Biosystems, Inc., Mississauga, OR, Canada). Briefly, the DNA fragment for murine CD38 was amplified from the splenocytes $\left(1 \times 10^{6}\right.$ cells) of C57BL/6 mice by polymerase chain reaction (PCR) using the following specific primers: Sense, 5'-GGGGTACCTTCGGGAGCCCAATGGCTAA-3' and antisense, 5'-GCTCTAGAAGTCCAGGCTACAGGTGAT CTAA-3'. The primers were synthesized by Sangon Biotech (Shanghai) Co., Ltd. (Shanghai, China). The PCR was performed under the following conditions: $5 \mathrm{~min}$ at $94^{\circ} \mathrm{C} ; 30$ cycles for 45 sec at $94^{\circ} \mathrm{C}, 45 \mathrm{sec}$ at $55^{\circ} \mathrm{C}$, and $90 \mathrm{sec}$ at $72^{\circ} \mathrm{C}$, and ended with $10 \mathrm{~min}$ at $72^{\circ} \mathrm{C}$. The $\mathrm{CD} 38$ sequence was subsequently inserted into a pDC315 shuttle vector (BioVector, Beijing, China) and then $5 \mu \mathrm{g}$ pDC315 vector was mixed with $5 \mu \mathrm{g}$ pBHGlox $\Delta \mathrm{E} 1,3 \mathrm{Cre}$ Ad backbone plasmid (BioVector), $10 \mu$ l Lipofectamine 2000 (Thermo Fisher Scientific, Inc.). The mixture was transfected into HEK293 cells (American Type Culture Collection, Manassas, VA, USA). Approximately 10-15 days later, the cells floated and were collected in $1 \mathrm{ml}$ phosphate-buffered saline (PBS). Then the cells were subjected to 3 cycles of freeze-thaw and the cell debris was removed by centrifugation $(12,000 \mathrm{x} \mathrm{g}$ for $15 \mathrm{~min}$ ). The supernatants were then rich in Ad-CD38, and were stored at $-80^{\circ} \mathrm{C}$ until further use.

Generation of bone marrow-derived DCs (BMDCs) and Ad infection. After removing all muscle tissues with gauze from the femurs and tibias, the bones were placed in a $60-\mathrm{mm}$ dish with $70 \%$ alcohol for $1 \mathrm{~min}$, washed twice with PBS, and transferred into a fresh dish with RPMI 1640. Both ends of the bones were cut with scissors in the dish, and then the marrow 
was flushed out using $2 \mathrm{ml}$ RPMI 1640 with a syringe and 25 -gauge needle. The tissue was suspended, passed through nylon mesh to remove small pieces of bone and debris, and red cells were lysed with ammonium chloride. Then the bone marrow mononuclear cells were cultured at a density of $2 \times 10^{6}$ cells/ml in RPMI 1640 medium supplemented with $10 \%$ fetal calf serum (Thermo Fisher Scientific, Inc., Waltham, MA, USA), $10 \mathrm{ng} / \mathrm{ml} \mathrm{rmGM-CSF}$ and $1 \mathrm{ng} / \mathrm{ml} \mathrm{IL-} 4$ at $37^{\circ} \mathrm{C}$. Non-adherent cells were gently washed away after $48 \mathrm{~h}$ of culture; the remaining loosely adherent clusters were cultured for a further $48 \mathrm{~h}$ and were harvested for Ad transduction. For Ad infection, $1 \times 10^{6}$ BMDCs were mixed with $5 \times 10^{7}$ PFU Ad in a total volume of $1 \mathrm{ml}$ serum-free medium for $24 \mathrm{~h}$.

Fluorescence-activated cell sorting (FACS) analysis. To confirm CD38 expression, Ad-infected BMDCs were stained with anti-CD38 at $4^{\circ} \mathrm{C}$ for $20 \mathrm{~min}$. Ad-infected BMDCs were stimulated with or without $100 \mathrm{ng} / \mathrm{ml} \mathrm{LPS}$ for $24 \mathrm{~h}$ at $37^{\circ} \mathrm{C}$. The BMDCs were then collected and stained with MHC-II, CD80, $\mathrm{CD} 86$ and CD40 antibodies at $4^{\circ} \mathrm{C}$ for $20 \mathrm{~min}$. The non-specific binding of BMDCs and antibodies was determined by isotype control antibodies staining at $4^{\circ} \mathrm{C}$ for $20 \mathrm{~min}$. For the induction of Th1 cell differentiation, $\mathrm{CD}^{+}{ }^{+}$naïve $\mathrm{T}$ cells from splenocytes were purified using the Mouse Naïve $\mathrm{CD}^{+}{ }^{+} \mathrm{T}$ cell Isolation kit according to the manufacturers instructions. Subsequently, $2 \times 10^{6} / \mathrm{ml}$ naïve $\mathrm{CD}^{+}{ }^{+} \mathrm{T}$ cells were seeded into $1 \mu \mathrm{g} / \mathrm{ml}$ anti-CD3 (BE0002; Bio X Cell) and $1 \mu \mathrm{g} / \mathrm{ml}$ anti-CD28 (BE0015-1; Bio X Cell) pre-coated 96-well plates in the presence of $10 \mu \mathrm{g} / \mathrm{ml}$ anti-IL-4. Ad-CD38-infected BMDCs (Ad-CD38/BMDCs), Ad-LacZ-infected BMDCs (Ad-LacZ/BMDCs) or equal volume PBS-treated BMDCs (Control/BMDCs) were added at a ratio of 10:1. Ad-LacZ was donated by Dr Zhijian Cai (Zhejiang University). $\mathrm{CD}^{+}$naïve $\mathrm{T}$ cells co-cultured with each group of BMDCs without anti-CD3 and anti-CD28 stimulation were designated as Th0 cells. After 3 days of induction at $37^{\circ} \mathrm{C}$, the cells were collected and stimulated with phorbol 12-myristate 13-acetate/ionomycin for $5 \mathrm{~h}$. The cells were then stained with anti-CD 4 antibody at $4^{\circ} \mathrm{C}$ for $20 \mathrm{~min}$, were fixed and permeabilized using the Intracellular Fixation \& Permeabilization Buffer set, and were stained with anti-IFN- $\gamma$ antibody at $4{ }^{\circ} \mathrm{C}$ for $20 \mathrm{~min}$. All cells were examined by flow cytometry, and data were analyzed using the FlowJo software, version 7.6 (FlowJo, LLC, Ashland, OR, USA).

Quantitative $(q) P C R$. Th1 cells were induced as aforementioned. After 3 days of coculture with BMDCs, $\mathrm{CD}^{+} \mathrm{T}$ cells were sorted and total RNA was extracted from the $\mathrm{CD}^{+}$ T cells using TRIzol ${ }^{\circledR}$ reagent (Invitrogen; Thermo Fisher Scientific, Inc.) according to the manufacturer's protocol. The RNA was then reverse transcribed into cDNA using ReverTra Ace qPCR RT Kit (FSQ-101; Toyobo Co., Ltd., Osaka, Japan) according to the manufacturer's protocol and the qPCR was performed using SYBR Premix Ex Taq (RR420A; Takara Bio, Inc., Kusatsu, Japan) in a final volume of $20 \mu 1$ according to the manufacturer's protocol. The specific primers synthesized by Sangon Biotech (Shanghai) Co., Ltd. used for qPCR were: $\beta$-actin, sense 5'-CGTTGACATCCGTAAAGACC-3', antisense 5'-AACAGTCCGCCTAGAAGCAC-3'; T-bet, sense 5'-AGC AAGGACGGCGAATGTT-3', antisense 5'-GGGTGGACA TATAAGCGGTTC-3'; and IFN- $\gamma$, sense 5'-AGCGGCTGA
CTGAACTCAGATTGTAG-3' and antisense 5'-GTCACAGTT TTCAGCTGTATAGGG-3'. The following PCR conditions were used: 1 cycle at $95^{\circ} \mathrm{C}$ for $30 \mathrm{sec}$, followed by 40 cycles at $95^{\circ} \mathrm{C}$ for $5 \mathrm{sec}$ and $60^{\circ} \mathrm{C}$ for $34 \mathrm{sec}$. qPCR was performed using an Applied Biosystems 7500 Real Time PCR system (Applied Biosystems; Thermo Fisher Scientific, Inc.). The relative mRNA levels of the target gene were calculated by the following formula: 2(Ct of target gene - $\mathrm{Ct}$ of $\beta$-actin). The relative mRNA levels were normalized by the relative mRNA level of one sample from the Control/BMDCs + LPS group.

Protocol for the model of allergen-challenged mice. Mice were sensitized on days 0 and 14 by intraperitoneal injection of $0.08 \mathrm{mg}$ OVA and $0.1 \mathrm{ml}$ aluminum hydroxide in $0.1 \mathrm{ml}$ PBS or PBS alone. A total of $1 \times 10^{6}$ Ad-infected BMDCs were intravenously injected into the OVA-sensitized mice on day 23. Subsequently, OVA- or PBS-sensitized mice were exposed to aerosolized 1\% OVA/0.01\% LPS/PBS or normal saline for $40 \mathrm{~min}$, respectively, once per day for 3 consecutive days (days 24-26). On day 27, the mice were sacrificed by intraperitoneal injection with nembutal (160 mg/kg) and the lungs were divided into two groups for analysis: The left lung lobes were lavaged three times with $1 \mathrm{ml}$ PBS containing $1 \%$ fetal calf serum and $5 \mathrm{U} / \mathrm{ml}$ heparin, and the right lung lobes were fixed with $4 \%$ paraformaldehyde for hematoxylin $(0.5 \% ; 5 \mathrm{~min})$ and eosin $(1 \% ; 30 \mathrm{sec})$ and Periodic acid $(0.1 \% ; 10 \mathrm{~min})$ and Schiff $(0.5 \% ; 10 \mathrm{~min})$ Schiff staining. Imaging was conducted using the BX53 microscope (Olympus Corporation, Tokyo, Japan).

Cytokine assays. Ad-infected BMDCs were stimulated with or without $100 \mathrm{ng} / \mathrm{ml}$ LPS for $24 \mathrm{~h}$. To collect sera, mice were anesthetised by intraperitoneal injection with nembutal $(80 \mathrm{mg} / \mathrm{kg})$. Blood was exsanguinated from the heart with a syringe and 25-gauge needle and sera were collected after incubation at room temperature for $30 \mathrm{~min}$. IL-12 levels in the supernatant were detected by ELISA. The levels of IL-4, IL-5, IL-13 and IFN- $\gamma$ in the bronchoalveolar lavage fluid (BALF), and the levels of IgE in the sera were also detected by ELISA. Th1 cells were induced as aforementioned. After 3 days of coculture with BMDCs, $\mathrm{CD}^{+} \mathrm{T}$ cells were sorted and re-stimulated with $1 \mu \mathrm{g} / \mathrm{ml}$ anti-CD3 and $1 \mu \mathrm{g} / \mathrm{ml}$ anti-CD28 (pre-coated onto plates) for $24 \mathrm{~h}$. Subsequently, the levels of IFN- $\gamma$ in the supernatant were measured by ELISA. All ELISA assays were conducted according to manufacturer's instructions.

Western blot analysis. For the detection of p-p38, Ad-infected BMDCs were stimulated with $100 \mathrm{ng} / \mathrm{ml}$ LPS for the indicated duration. Subsequently, the cells were washed and lysed by RIPA lysis buffer (Beyotime Institute of Biotechnology, Haimen, China). The protein concentrations of lysates were measured by the Bradford assay kit (Thermo Fisher Scientific, Inc.). Cell proteins $(20 \mu \mathrm{g})$ were separated by $10 \%$ sodium dodecyl sulfate-polyacrylamide gel electrophoresis, and were transferred onto polyvinylidene difluoride membranes. Membranes were blocked by Tris-buffered saline with Tween-20 containing $5 \%$ bovine serum albumin (Sigma-Aldrich; Merck Millipore) at room temperature for $2 \mathrm{~h}$ and then incubated with p-p38 $(1: 1,000)$ and p38 $(1: 1,000)$ primary antibodies at room temperature for $1 \mathrm{~h}$, followed by horseradish peroxidase-coupled secondary antibody (sc-2005; 1:500) at room temperature for $1 \mathrm{~h}$. Specific 
A

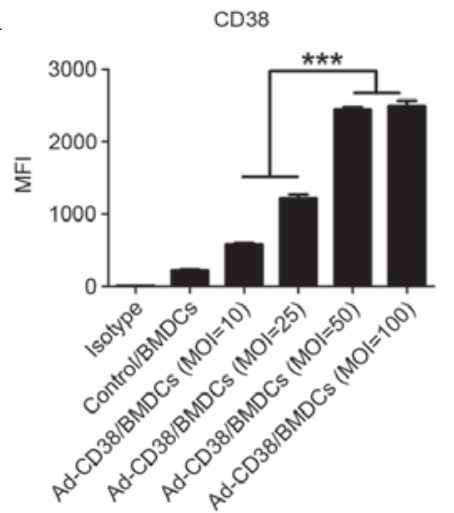

B

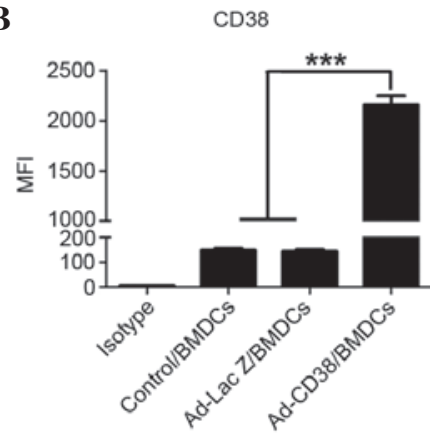

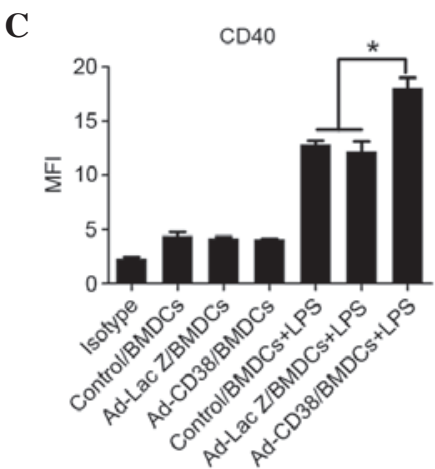
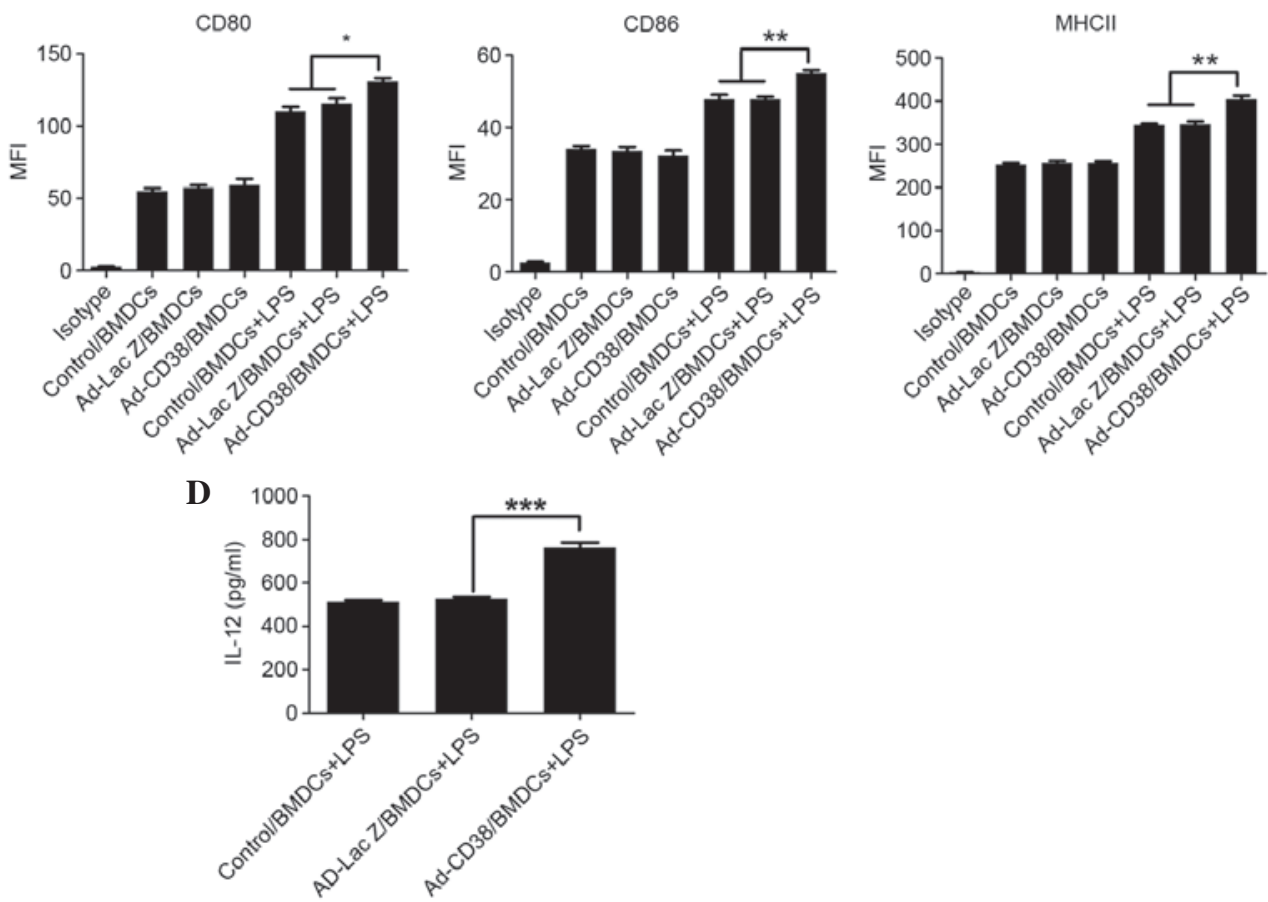

Figure 1. CD38 promotes LPS-induced maturation of BMDCs and IL-12 secretion. (A) BMDCs were infected with Ad-CD38 at an MOI of $10,25,50$ or 100 for $24 \mathrm{~h}$. CD38 expression in BMDCs was detected by FACS. (B-D) BDMCs were infected with Ad-CD38 (MOI 50 ) Ad-LacZ or were mock infected for $24 \mathrm{~h}$. (B) CD38 expression in these BMDCs was detected by FACS. (C) Ad-infected BMDCs were stimulated with or without 100 ng/ml LPS for 24 h. MHC-II, CD80, CD86 and CD40 expression was detected by FACS ( $\mathrm{n}=3$ ). (D) IL-12 production in the supernatants was detected by enzyme-linked immunosorbent assay $(\mathrm{n}=5)$. Data are representative of three independent experiments. ${ }^{*} \mathrm{P}<0.05,{ }^{* *} \mathrm{P}<0.01,{ }^{* * *} \mathrm{P}<0.001$. CD, cluster of differentiation; LPS, lipopolysaccharide; BMDCs, bone marrow-derived dendritic cells; IL, interleukin; MOI, multiplicity of infection; FACS, fluorescence-activated cell sorting; Ad, adenovirus; MHC-II, major histocompatibility complex class II; MFI, mean fluorescent intensity.

bands on the membrane were then visualized using an enhanced chemiluminescence (ECL) kit (ECL Detection kit; Amersham Biosciences; GE Healthcare Life Sciences, Little Chalfont, UK).

Statistical analysis. Data are presented as the mean \pm standard error of the mean. The significance of differences between groups was estimated using the unpaired Student's t-test for two groups, or one-way analysis of variance followed by the Newman-Keuls test for multiple group comparisons. GraphPad Prism softtware, version 5 (GraphPad, Inc., La Jolla, CA, USA) was used for statistical analysis. $\mathrm{P}<0.05$ was considered to indicate a statistically significant difference.

\section{Results}

CD38 promotes LPS-induced maturation of DCs and IL-12 secretion. The number of natural DCs is very limited, therefore,
BMDCs are widely used for study of DC function in vitro and in vivo. CD38 was overexpressed in BMDCs following Ad-CD38 infection. To confirm the optimal multiplicity of infection (MOI), BMDCs were infected with Ad-CD38 at the following MOI: 10, 25, 50 and 100. A total of 24 hours after infection, CD38 expression was detected in BMDCs by FACS. As MOI increased, CD38 expression in BMDCs was elevated. However, there was no difference in CD38 expression between BMDCs infected with an MOI of 50 or 100 (Fig. 1A). Therefore, for subsequent experiments, MOI 50 was selected for BMDC infection. Subsequently, CD38 expression was compared between Ad-CD38/BMDCs, Ad-LacZ/BMDCs or Control/BMDCs. The results demonstrated that Ad-CD38/BMDCs, but not Ad-LacZ/BMDCs or Control/BMDCs, exhibited increased CD38 expression (Fig. 1B). By detecting the expression levels of MHC-II, CD80, CD86 and CD40 molecules, overexpression of CD38 was not able to affect DC maturation. However, following LPS stimulation, a more 


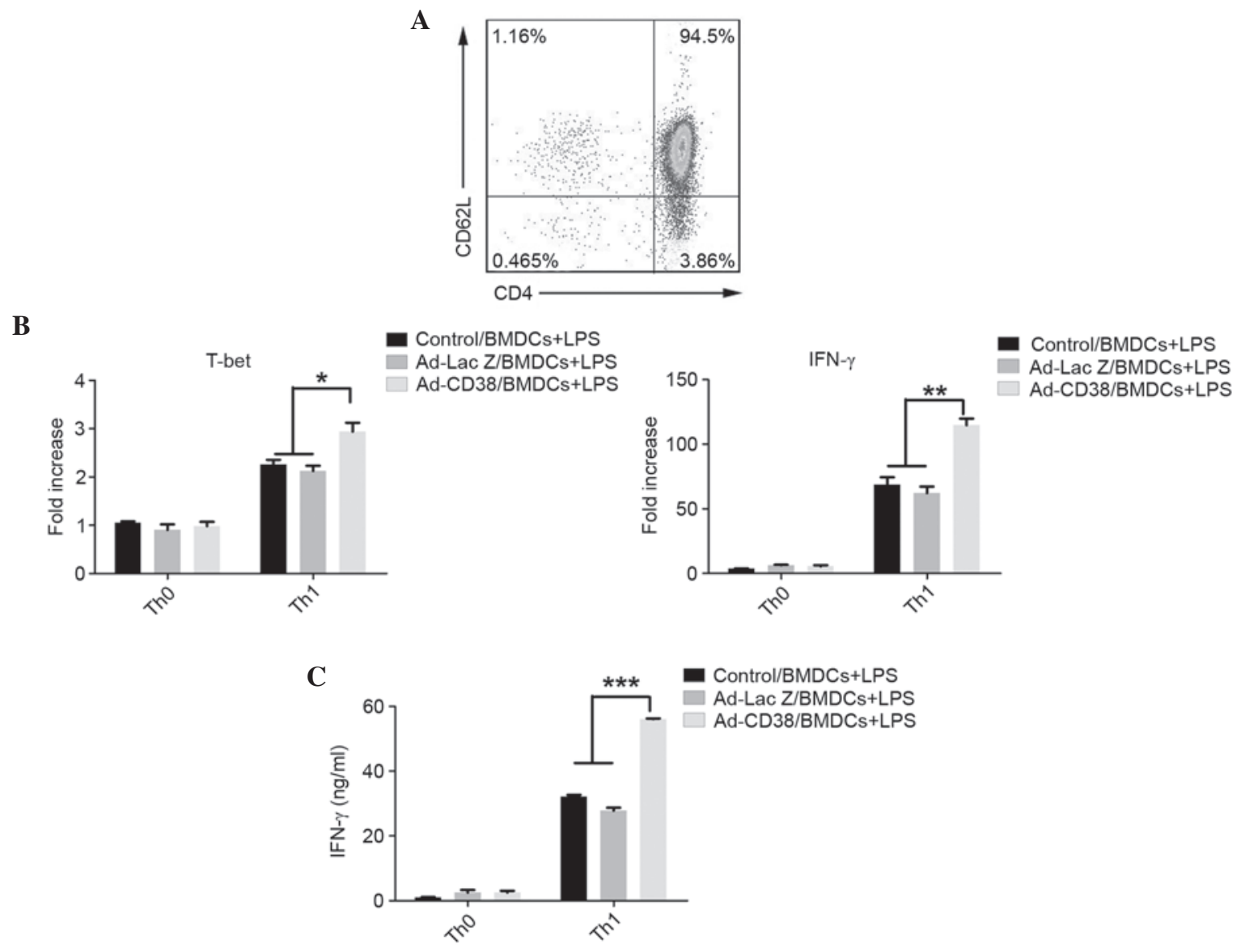

D
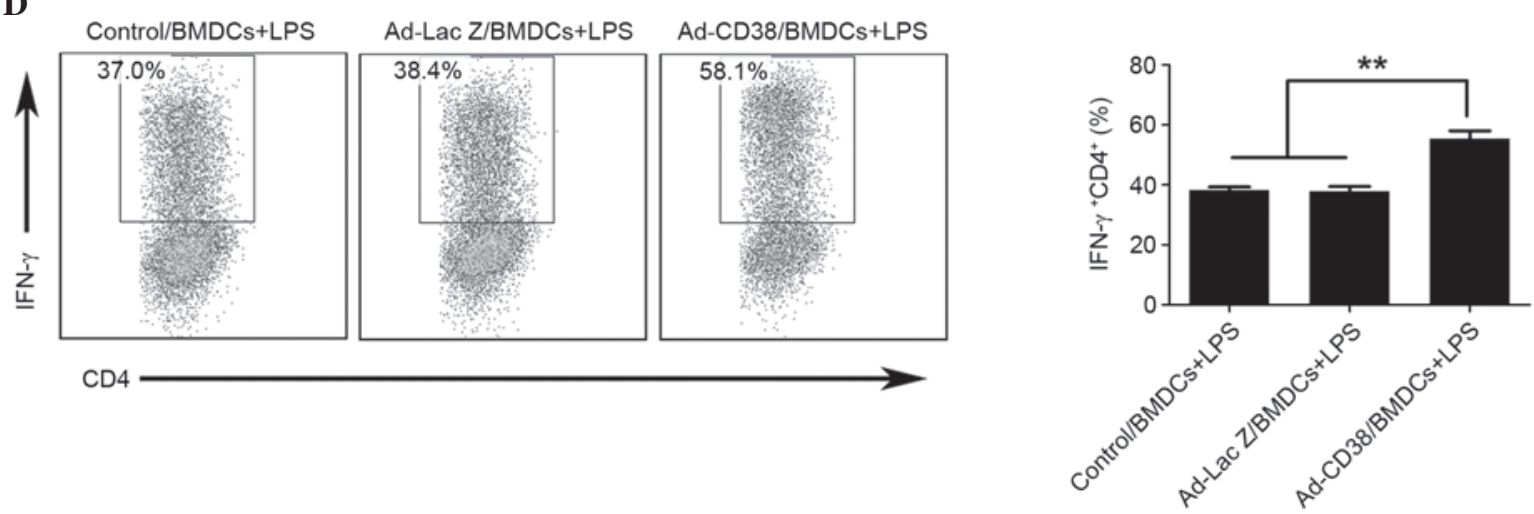

Figure 2. LPS-stimulated Ad-CD38/BMDCs promote Th1 differentiation. (A) Naïve CD4+ T cells were isolated and purity was confirmed by FACS. (B-D) A total of $2 \times 10^{6} / \mathrm{ml}$ naïve CD4 ${ }^{+} \mathrm{T}$ cells were seeded into $1 \mu \mathrm{g} / \mathrm{ml}$ anti-CD3 and $1 \mu \mathrm{g} / \mathrm{ml}$ anti-CD28 pre-coated 96 -well plates in the presence of $10 \mu \mathrm{g} / \mathrm{ml}$ anti-IL-4. Meanwhile, each group of BMDCs was added at a ratio of 10:1. After 3 days of induction, $\mathrm{CD}^{+} \mathrm{T}$ cells were isolated. (B) The mRNA expression levels of IFN- $\gamma$ and T-bet were detected in CD4 ${ }^{+} \mathrm{T}$ cells by quantitative polymerase chain reaction. (C) $\mathrm{CD} 4{ }^{+} \mathrm{T}$ cells were re-stimulated with $1 \mu \mathrm{g} / \mathrm{ml}$ anti-CD3 and $1 \mu \mathrm{g} / \mathrm{ml}$ anti-CD28 (pre-coated onto plates) for $24 \mathrm{~h}$ and the protein expression levels of IFN- $\gamma$ were detected in the supernatant by enzyme-linked immunosorbent assay. (D) After 3 days of induction, the percentage of IFN- $\gamma^{+} \mathrm{CD} 4^{+} \mathrm{T}$ cells was detected by FACS following intracellular staining with IFN- $\gamma$. Data are representative of three independent experiments, each with $\mathrm{n}=3 .{ }^{*} \mathrm{P}<0.05,{ }^{* *} \mathrm{P}<0.01,{ }^{* * * *} \mathrm{P}<0.001$. LPS, lipopolysaccharide; Ad, adenovirus; CD, cluster of differentiation; BMDCs, bone marrow-derived dendritic cells; Th1, T helper 1; FACS, fluorescence-activated cell sorting; IL, interleukin.

mature phenotype of Ad-CD38/BMDCs was detected compared with the Ad-LacZ/BMDCs and Control/BMDCs (Fig. 1C). The levels of IL-12 in Ad-CD38/BMDCs, Ad-Lac Z/BMDCs and Control/BMDCs were low and showed no difference without LPS stimulation (data not shown). Similarly, a higher production of IL-12 could only be detected in Ad-CD38/BMDCs following LPS stimulation (Fig. 1D). These results suggest that CD38 synergizes with LPS to promote DC maturation and IL-12 secretion.
LPS-stimulated Ad-CD38/BMDCs promote Th1 differentiation. Since Ad-CD38/BMDCs stimulated with LPS produced more IL-12, the present study aimed to determine whether Ad-CD38/BMDCs were more effective at inducing Th1 cell differentiation. Naïve $\mathrm{CD}^{+} \mathrm{T}$ cells were isolated using the Naïve $\mathrm{CD}^{+} \mathrm{T}$ cell Isolation kit, and the purity of naïve $\mathrm{CD}^{+} \mathrm{T}$ cells was $\sim 95 \%$ (Fig. $2 \mathrm{~A}$ ). Naïve $\mathrm{CD} 4^{+} \mathrm{T}$ cells were cocultured with BMDCs at a ratio of 10:1 for 3 days in Th1 cell-skewing conditions; with the exception of IL-12. 
$\mathbf{A}$

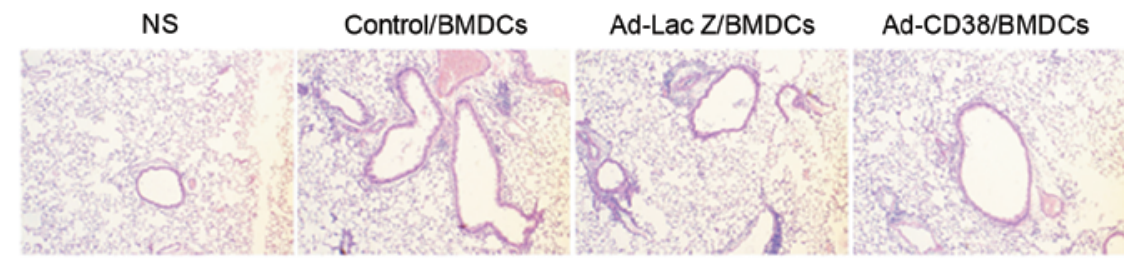

PAS

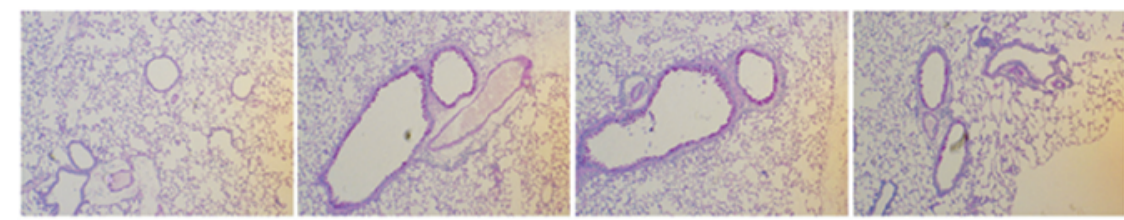

B
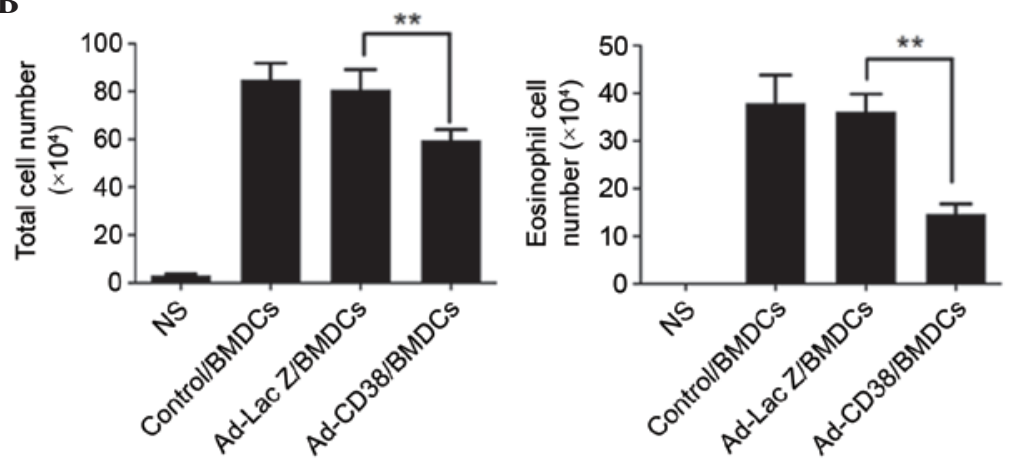

C
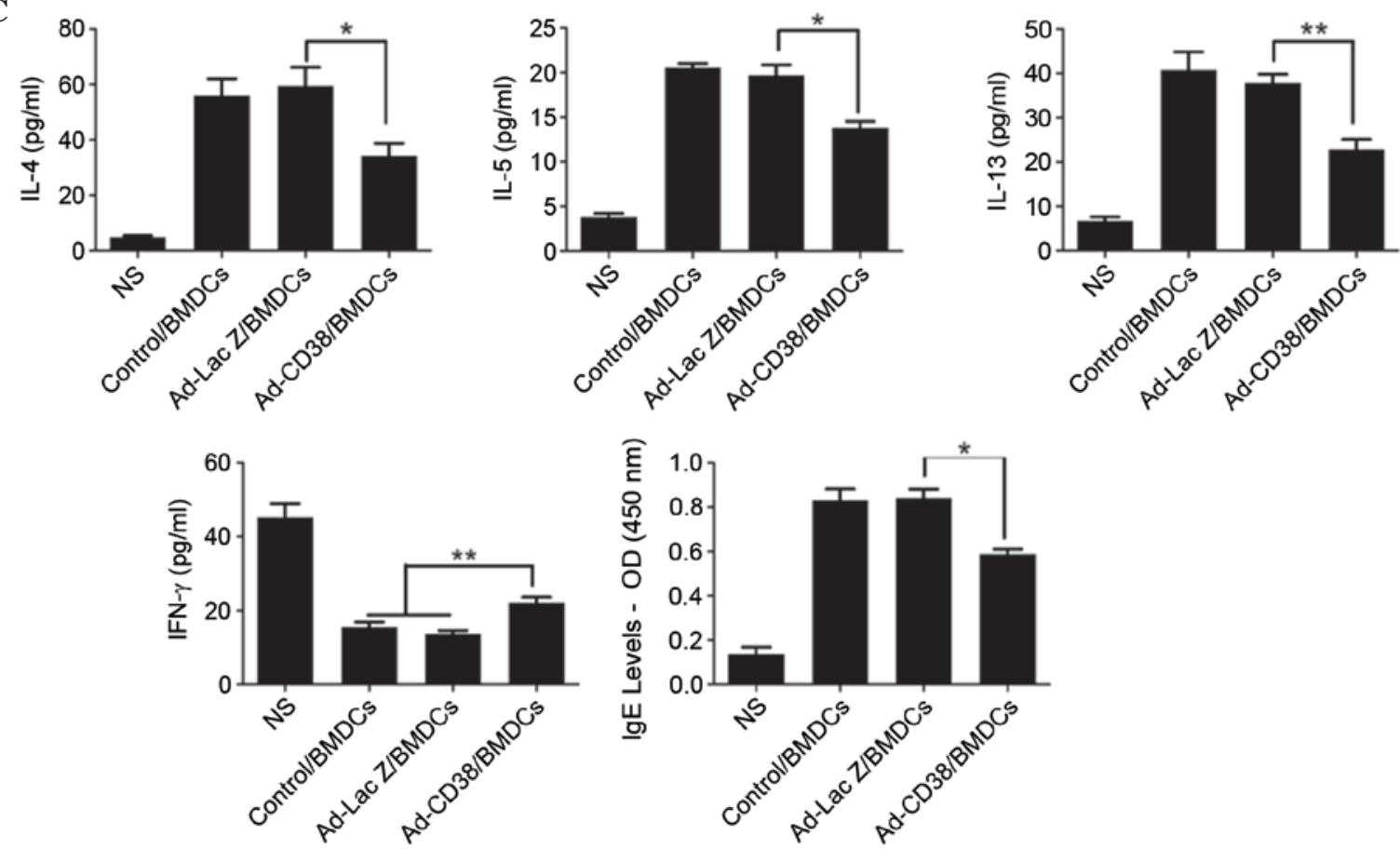

Figure 3. Administration of Ad-CD38/BMDCs can alleviate the severity of murine asthma. Mice were sensitized with PBS or OVA plus aluminium hydroxide on days 0 and 14. On day 23, 1x10 ${ }^{6}$ Control/BMDCs, Ad-LacZ/BMDCs or Ad-CD38/BMDCs were intravenously injected into OVA-sensitized mice (6 mice/group). The mice were then challenged with aerosolized PBS containing 1\% OVA and 0.01\% LPS (OVA-sensitized mice) or challenged with aerosolized NS (PBS-sensitized mice) for 3 consecutive days (days 24-26). Mice were sacrificed $24 \mathrm{~h}$ after the last challenge and the lungs were isolated. (A) Formalin-fixed lung tissue sections were stained with H\&E or PAS (magnification, $x 400$ ). (B) Total cells and eosinophils in the BALF were enumerated. (C) Levels of IL-4, IL-5, IL-13 and IFN- $\gamma$ in the BALF, and IgE in the sera were measured by enzyme-linked immunosorbent assay. Data are representative of three independent experiments, each with $\mathrm{n}=5 .{ }^{*} \mathrm{P}<0.05,{ }^{* *} \mathrm{P}<0.01$. Ad, adenovirus; $\mathrm{CD}$, cluster of differentiation; BMDCs, bone marrow-derived dendritic cells; PBS, phosphate-buffered saline; LPS, lipopolysaccharide; NS, normal saline; OVA, ovalbumin; BALF, bronchoalveolar lavage fluid; IL, interleukin; IFN- $\gamma$, interferon- $\gamma$; IgE, immunoglobulin E; H\&E, hematoxylin and eosin; PAS, Periodic acid-Schiff; OD, optical density.

As expected, the mRNA expression levels of IFN- $\gamma$ and T-bet, and the protein levels of IFN- $\gamma$ were higher in $\mathrm{CD}^{+}$ $\mathrm{T}$ cells cocultured with LPS-stimulated Ad-CD38/BMDCs, as compared with in $\mathrm{CD} 4^{+} \mathrm{T}$ cells cocultured with LPS-stimulated Ad-LacZ/BMDCs or Control/BMDCs (Fig. 2B and C). To further confirm these results, IFN- $\gamma$ 
A

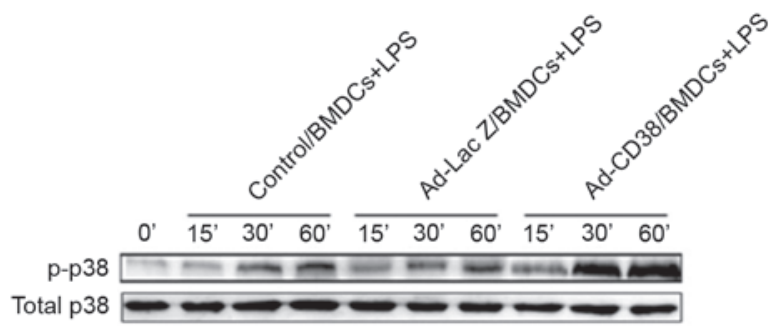

C

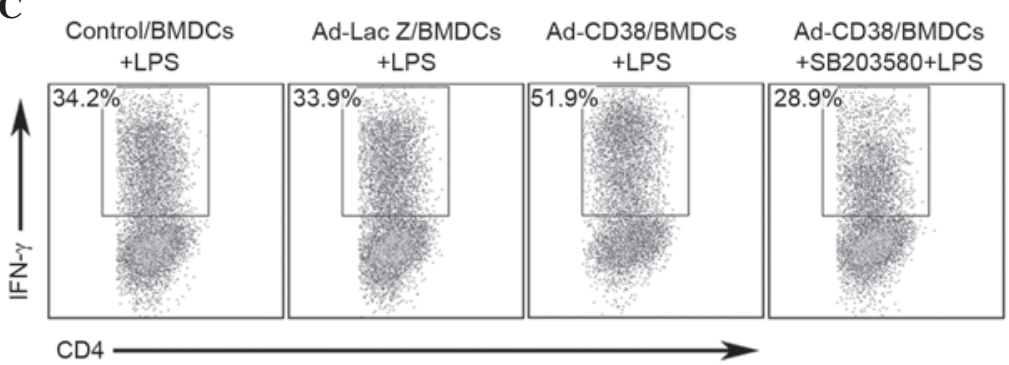

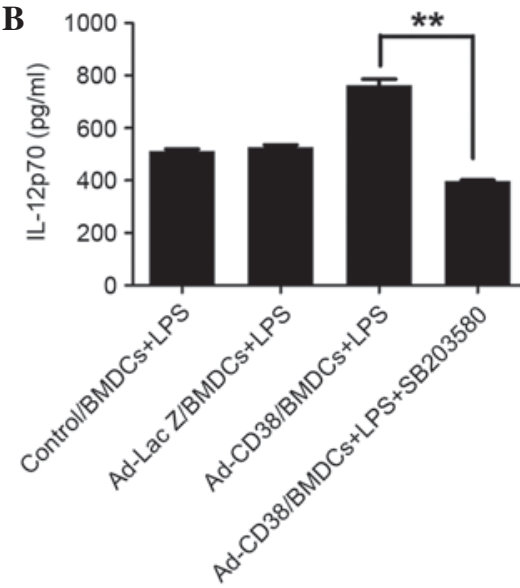

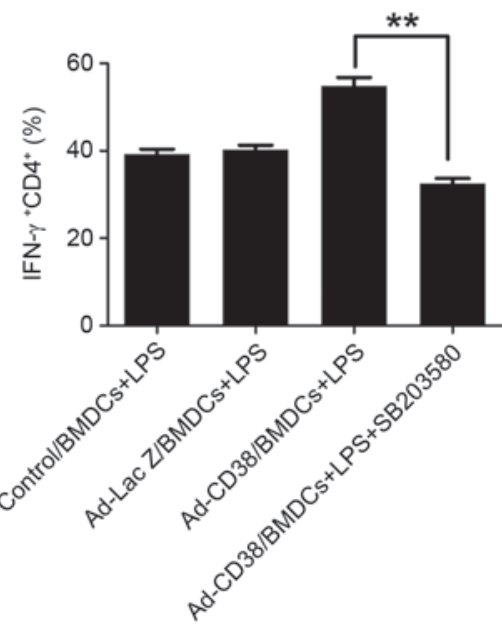

Figure 4. CD38 promotes LPS-induced BMDCs to release IL-12 and induce Th1 differentiation in a p38-dependent manner. BMDCs were transduced with Ad-CD38 (multiplicity of infection 50) or Ad-LacZ, or were mock-infected for $24 \mathrm{~h}$. (A) These BMDCs were stimulated with or without 100 ng/ml LPS for the indicated time. The expression levels of p-p38 were detected by western blotting. (B and C) BMDCs were then pre-treated with or without 50 nM SB203580 for $30 \mathrm{~min}$ and were stimulated with $100 \mathrm{ng} / \mathrm{ml}$ LPS for $24 \mathrm{~h}$. (B) IL-12p70 production in the supernatants was detected by enzyme-linked immunosorbent assay $(n=5)$. (C) Th1 cell differentiation was induced. After 3 days of induction, the percentage of IFN- $\gamma^{+}$CD $4^{+}$T cells was detected by FACS following intracellular staining of IFN $-\gamma(n=3)$. Data are representative of three independent experiments. ${ }^{* *} \mathrm{P}<0.01$. CD, cluster of differentiation; LPS, lipopolysaccharide; BMDCs, bone marrow-derived dendritic cells; Ad, adenovirus; p-, phosphorylated; Th1, T helper 1; FACS, fluorescence-activated cell sorting; IFN- $\gamma$, interferon- $\gamma$.

levels were measured by intracellular staining of IFN- $\gamma$ in $\mathrm{CD}^{+} \mathrm{T}$ cells. As shown in Fig. 2D, the highest percentage of IFN- $\gamma^{+} \mathrm{CD} 4^{+} \mathrm{T}$ cells was detected in $\mathrm{CD} 4^{+} \mathrm{T}$ cells cocultured with LPS-stimulated Ad-CD38/BMDCs (Fig. 2D). These results indicate that LPS-stimulated Ad-CD38/BMDCs exhibit a higher potential for inducing Th1 cell differentiation.

Ad-CD38/BMDCs can alleviate the severity of murine asthma. To determine whether Ad-CD38/BMDCs exert protective effects on murine asthma, $1 \times 10^{6}$ Ad-infected BMDCs were intravenously injected into OVA antigen-immunized mice $24 \mathrm{~h}$ prior to the first OVA antigen challenge. After three OVA challenges, the recipients of Control/BMDCs, Ad-LacZ/BMDCs and Ad-CD38/BMDCs all developed airway inflammation, which was characterized by goblet cell hyperplasia, peribronchovascular eosinophilic infiltration, increased production of mucus, and a large number of total cells and eosinophil fractions in the BALF (Fig. 3A and B). However, the recipients of Ad-CD38/BMDCs exhibited the mildest symptoms of asthma (Fig. 3A and B). In addition, production of IL-4, IL-5 and IL-13 was increased in the BALF, increased IgE was detected in the sera, and production of IFN- $\gamma$ was reduced in the BALF of recipients of Control/BMDCs and Ad-LacZ/BMDCs (Fig. 3C). These results suggest that Ad-CD38/BMDCs may inhibit asthma development in vivo, probably through regulating the Th1/Th2 cell balance.

CD38 is dependent on the p38 signaling pathway to promote LPS-induced BMDCs to release IL-12 and induce Thl differentiation. It has previously been reported that activation of the p38 signaling pathway is involved in IL-12 secretion by macrophages (24). The present study determined whether CD38 promoted LPS-induced BMDCs to secrete IL-12 via the p38 signaling pathway. Following LPS stimulation, the expression levels of p-p38 were increased in Control/BMDCs, Ad-LacZ/BMDCs and Ad-CD38/BMDCs; the biggest increase was detected in Ad-CD38/BMDCs (Fig. 4A). To further confirm the p38 signaling pathway was associated with increased IL-12 production in LPS-stimulated Ad-CD38/BMDCs, BMDCs were pre-treated with a p38-specific inhibitor, SB203580. The results demonstrated that there was no significant 
difference in IL-12 production between the Control/BMDCs, Ad-LacZ/BMDCs and Ad-CD38/BMDCs following LPS stimulation (Fig. 4B). However, following pretreatment of BMDCs with SB203580, IL-12 secretion was decreased and the increased induction of Th1 cell differentiation by Ad-CD38/BMDCs was abrogated (Fig. 4B and C). These results suggest that LPS-stimulated Ad-CD38/BMDCs depend on the p38 signaling pathway to promote IL-12 production and Th1 cell differentiation.

\section{Discussion}

It is well known that a dysregulated Th1/Th2 balance is the predominant mechanism underlying asthma development. The present study demonstrated that overexpression of CD38 synergized with LPS to induce IL-12 secretion from BMDCs and Th1 differentiation. CD38-overexpressing BMDCs were effective at alleviating the severity of asthma, which was accompanied by a decrease in the Th2 master cytokine IL-4, and an increase in the Th1 master cytokine IFN- $\gamma$ in the BALF of asthmatic mice. These results suggested that CD38 may be involved in the regulation of asthma development via its effects on the Th1/Th2 balance.

Consistent with the results of a previous study, CD38 is able to synergize with LPS to induce IL-12 secretion from DCs (25). However in this previous study, the authors did not elucidate whether activation of the CD38 signal alone could promote IL-12 secretion from DCs. According to the results of the present study, the CD38 signal alone exhibited no effect on IL-12 secretion of DCs. The CD38 signal is more inclined to regulate LPS-induced signal activation in DCs, particularly via the p38 signaling pathway. As LPS is the natural ligand for Toll-like receptor (TLR) 4 (26), it is suggested that there is a point of intersection between TLR4 signaling and the CD38 signal. Unlike in vitro results, administration of Ad-CD38/BMDCs, without LPS stimulation, was able to alleviate asthmatic symptoms in vivo. The TLR4 signal in DCs has been reported to be activated in asthmatic mice (27). TLR4 signaling in Ad-CD38/BMDCs may also be activated in asthmatic mice, and CD38 signaling may possibly further promote activation of TLR4 signaling in Ad-CD38/BMDCs.

CD31 is a platelet endothelial cell adhesion molecule, which is a member of the immunoglobulin superfamily and is considered the counter-receptor of CD38. DCs express CD31 and CD38 at the same time (data not shown), so they may activate CD38 signal by themselves. In addition, CD31 has been demonstrated to be overexpressed in endothelial cells $(28,29)$, suggesting CD38 signals in DCs may also be activated by endothelial cells. Therefore, it is reasonable to suggest that CD38 signaling in DCs is easily activated in vivo, thus indicating the important function of CD38 in DCs.

In conclusion, the present study demonstrated that overexpression of CD38 in BMDCs was able to increase LPS-induced IL-12 secretion and promote Th1 cell differentiation in a p38 signaling pathway-dependent manner. CD38-overexpressing BMDCs exhibited protective effects on murine asthma, potentially via restoration of the Th1/Th2 balance. Therefore, CD38 may be considered a promising candidate for the treatment of asthma.

\section{Acknowledgements}

The present study was supported by the National Natural Science Foundation of China (grant nos. 81200014 and 81300203), the Public Welfare Technology Application Research Project of Zhejiang Province (grant no. 2013C33146) and the Medicine and Health Foundation of the Health Bureau of Zhejiang Province (grant no. 2012KYA152).

\section{References}

1. D'Amato G, Holgate ST, Pawankar R, Ledford DK, Cecchi L, Al-Ahmad M, Al-Enezi F, Al-Muhsen S, Ansotegui I, Baena-Cagnani CE, et al: Meteorological conditions, climate change, new emerging factors and asthma and related allergic disorders. A statement of the world allergy organization. World Allergy Organ J 8: 25, 2015.

2. Sun R, Xu F, Wang C and Dong E: NSFC spurs significant basic research progress of respiratory medicine in China. Clin Respir J, 2015 (Epub ahead of print).

3. Barnes PJ: Glucocorticoids. Chem Immunol Allergy 100: 311-316, 2014.

4. Fajt ML and Wenzel SE: Asthma phenotypes and the use of biologic medications in asthma and allergic disease: The next steps toward personalized care. J Allergy Clin Immunol 135: 299-310; quiz 311, 2015.

5. Shen HH, Xu F, Zhang GS, Wang SB and Xu WH: CCR3 monoclonal antibody inhibits airway eosinophilic inflammation and mucus overproduction in a mouse model of asthma. Acta Pharmacol Sin 27: 1594-1599, 2006.

6. Wills-Karp M: Immunologic basis of antigen-induced airway hyperresponsiveness. Annu Rev Immunol 17: 255-281, 1999.

7. Neurath MF, Finotto S and Glimcher LH: The role of Th1/Th2 polarization in mucosal immunity. Nat Med 8: 567-573, 2002.

8. Bosnjak B, Stelzmueller B, Erb KJ and Epstein MM: Treatment of allergic asthma: Modulation of Th2 cells and their responses. Respir Res 12: 114, 2011.

9. Zhang Y, Zhang Y, Gu W, He L and Sun B: Th1/Th2 cell's function in immune system. Adv Exp Med Biol 841: 45-65, 2014.

10. Joffre O, Nolte MA, Spörri R and Reis e Sousa C: Inflammatory signals in dendritic cell activation and the induction of adaptive immunity. Immunol Rev 227: 234-247, 2009.

11. Hammad $\mathrm{H}$ and Lambrecht BN: Dendritic cells and airway epithelial cells at the interface between innate and adaptive immune responses. Allergy 66: 579-587, 2011.

12. Wu X and Xu F: Dendritic cells during Staphylococcus aureus infection: Subsets and roles. J Transl Med 12: 358, 2014.

13. Sung S, Rose CE and Fu SM: Intratracheal priming with ovalbumin- and ovalbumin 323-339 peptide-pulsed dendritic cells induces airway hyperresponsiveness, lung eosinophilia, goblet cell hyperplasia and inflammation. J Immunol 166: 1261-1271, 2001.

14. Heufler C, Koch F, Stanzl U, Topar G, Wysocka M, Trinchieri G, Enk A, Steinman RM, Romani N and Schuler G: Interleukin-12 is produced by dendritic cells and mediates $\mathrm{T}$ helper 1 development as well as interferon-gamma production by $\mathrm{T}$ helper 1 cells. Eur J Immunol 26: 659-668, 1996.

15. Koltsida O, Hausding M, Stavropoulos A, Koch S, Tzelepis G, Ubel C, Kotenko SV, Sideras P, Lehr HA, Tepe M, et al: IL-28A (IFN- $\lambda 2$ ) modulates lung DC function to promote Th1 immune skewing and suppress allergic airway disease. EMBO Mol Med 3: 348-361, 2011

16. Chen XQ, Yang J, Hu SP, Nie HX, Mao GY and Chen HB: Increased expression of CD86 and reduced production of IL-12 and IL-10 by monocyte-derived dendritic cells from allergic asthmatics and their effects on Th1- and Th2-type cytokine balance. Respiration 73: 34-40, 2006.

17. Malavasi F, Funaro A, Roggero S, Horenstein A, Calosso L and Mehta K: Human CD38: A glycoprotein in search of a function. Immunol Today 15: 95-97, 1994.

18. Jackson DG and Bell JI: Isolation of a cDNA encoding the human CD38 (T10) molecule, a cell surface glycoprotein with an unusual discontinuous pattern of expression during lymphocyte differentiation. J Immunol 144: 2811-2815, 1990. 
19. Deterre P, Berthelier V, Bauvois B, Dalloul A, Schuber F and Lund F: CD38 in T- and B-cell functions. Chem Immunol 75: $146-168,2000$.

20. Lande R, Urbani F, Di Carlo B, Sconocchia G, Deaglio S, Funaro A, Malavasi F and Ausiello CM: CD38 ligation plays a direct role in the induction of IL-1beta, IL-6, and IL-10 secretion in resting human monocytes. Cell Immunol 220: 30-38, 2002.

21. Zupo S, Rugari E, Dono M, Taborelli G, Malavasi F and Ferrarini M: CD38 signaling by agonistic monoclonal antibody prevents apoptosis of human germinal center B cells. Eur J Immunol 24: 1218-1222, 1994.

22. Viegas MS, do Carmo A, Silva T, Seco F, Serra V, Lacerda M and Martins TC: CD38 plays a role in effective containment of mycobacteria within granulomata and polarization of Th1 immune responses against Mycobacterium avium. Microbes Infect 9: 847-854, 2007.

23. Fedele G, Frasca L, Palazzo R, Ferrero E, Malavasi F and Ausiello CM: CD38 is expressed on human mature monocyte-derived dendritic cells and is functionally involved in CD83 expression and IL-12 induction. Eur J Immunol 34: 1342-1350, 2004.
24. Mathur RK, Awasthi A, Wadhone P, Ramanamurthy B and Saha B: Reciprocal CD40 signals through p38MAPK and ERK-1/2 induce counteracting immune responses. Nat Med 10: 540-544, 2004

25. Frasca L, Fedele G, Deaglio S, Capuano C, Palazzo R, Vaisitti T, Malavasi F and Ausiello CM: CD38 orchestrates migration, survival and Th1 immune response of human mature dendritic cells. Blood 107: 2392-2399, 2006.

26. Takeda K, Kaisho T, Akira S: Toll-like receptors. Annu Rev Immunol 21: 335-376, 2003.

27. Duez C, Gosset P and Tonnel AB: Dendritic cells and toll-like receptors in allergy and asthma. Eur J Dermatol 16: 12-16, 2006.

28. Deaglio S, Morra M, Mallone R, Ausiello CM, Prager E, Garbarino G, Dianzani U, Stockinger H and Malavasi F: Human CD38 (ADP-ribosyl cyclase) is a counter-receptor of CD31, an Ig superfamily member. J Immunol 160: 395-402, 1998.

29. Deaglio S, Dianzani U, Horenstein AL, Fernández JE, van Kooten C, Bragardo M, Funaro A, Garbarino G, Di Virgilio F, Banchereau J and Malavasi F: Human CD38 ligand. A 120-KDA protein predominantly expressed on endothelial cells. J Immunol 156: 727-734, 1996. 